\title{
Structure et typologie ichtyenne de trois bras morts de la Garonne1
}

\author{
D. Bengen 1 \\ A. Belaud 1 \\ P. Lim1
}

Mots clés : Garonne, bras morts, peuplements ichtyques, échantillonnage ponctuel d'abondance, analyse factorielle des correspondances, classification hièrarchique, typologie.

L'étude de la structure et de la typologie ichtyenne a été réalisée dans trois bras morts de la Garonne de 1988 à 1990. Les populations piscicoles ont été échantillonnées en utilisant deux méthodes de pêche complémentaires : l'Echantillonnage Ponctuel d'Abondance (E.P.A.) par pêche électrique et la pêche aux filets. Les échantillons obtenus par E.P.A. constituent la base de données principale pour cette étude.

Les peuplements ichtyques de ces trois bras morts sont dominés par les cyprinidés et présentent une forte similitude globale entre eux. Cette similitude, d'autant plus importante qu'il existe une communication plus large en aval avec le fleuve, traduit une forte interaction avec ce dernier et l'existence d'échanges migratoires fréquents.

Les espèces présentes dans les bras morts ont été séparées en groupes de tailles correspondant chacun aux principaux stades de développement. Il a été recherché les relations entre la présence de chaque espèce à chaque stade d'une part et, les caractéristiques de milieu et les phénomènes saisonniers d'autre part. L'analyse factorielle des correspondances conduit à distinguer trois types d'habitats (groupes spatials) possédant chacun des peuplements qui leur sont propres. La bouvière, ainsi que les juvéniles de grands cyprinidés préfèrent les zones peu profondes à proximité de végétation aquatique où se trouvent les substrats à granulométrie fine (limon-vase). Le second type d'habitat concerne surtout la végétation aquatique semi-immergée ; il accueille favorablement les especes carnassières de petites tailles (perche-soleil, perche et black-bass). Enfin, les rives à pente moyenne ou forte en bordure des zones profondes sur substrats graviers/galets, favorisent les populations d'adultes.

L'abondance de ces trois groupes de poissons évolue dans le temps selon trois grandes tendances: recrutement estival et automnal de juvéniles dans les bras morts, prépondérance hivernale d'individus immatures des cyprinidés et entrée d'adultes dans les bras morts au printemps.

\section{Fish population structure and typology in three ancients arms of the Garonne river}

Keywords : Garonne river, ancient arms, fish population, electro-fishing of spot samples, correspondence analysis, cluster analysis, typology.

A study of the structure and typology of the fish populations in three ancient arms of the Garonne river was performed during the period 1988 to 1990 . Fish were sampled using two complementary methods, regular abundance sampling using electro-fishing of spot samples (E.P.A.) and using nets. The samples obtained through E.P.A make up the bulk of the data used in this study.

The fish populations in the three ancient arms, which are generally similar, are dominated by the cyprinids. These ancient arms, all having direct access to the river, explain the important interactions and frequent migrations of populations from ancient arm to river and vice versa.

The species in these ancient arms are comprised of different size classes each one corresponding to the different stages of development of the fish. Relationships between the presence of each species at a certain life stage and seasonal changes in environmental characteristics were studied. A correspondence analysis between species interactions helped to identify three spatial groups (i.e., corresponding to habitats) of fish populations. The bitterlings as well as cyprinid juveniles prefer the shallow littoral zones with fine sand-mud substrates near aquatic vegetation. Zones with semi-submerged aquatic plants offer a favorable habitat for the small carnivorous species such as the sunfish and juveniles of perch and blackbass. Moderately sloped ancient arm banks and zones of deeper water along walled banks of gravel substrates favour adult populations.

Abundances of species from these three spatial groups depend upon the seasonal changes. However, three important tendencies had been identified, these are : summer and autumn recruitment of juveniles in the ancient arms, winter abundance of immature cyprinids and the preponderance of adults in spring.

1. Laboratoire d'Ichtyologie Appliquée, E.N.S.A. Toulouse, 145, Avenue de Muret, 31076 Toulouse Cedex, France. 


\section{Introduction}

Résultant d'une histoire géomorphologique, les principales caractéristiques du fleuve (pente, charge solide, débit) déterminent la puissance d'érosion qui creuse et élargit la plaine d'inondation. Dans ce lit élargi, se forment parfois des trains de méandres ou des chenaux multiples propices au recoupement et à l'abandon de chenaux par le cours actif. Ces méandres, qui progressent vers l'aval, occupent successivement toute la plaine alluviale (Décamps \& Naiman 1989). Au moment de la rupture de l'espace entre deux méandres, il peut subsister pendant plusieurs dizaines d'années des " bras morts " en communication plus ou moins importante et fréquente avec le fleuve. Ces bras morts sont particulièrement nombreux dans la vallée de la Garonne entre Toulouse et Montauban.

L'étude de bras morts similaires du Haut-Rhône a montré l'intérêt écologique de ces milieux en tant que zone-refuge pour la faune aquatique, notamment pour les populations piscicoles (Juget et al. 1976, Bouvet et al. 1982, Juget \& Roux 1982, Castella \& Amoros 1984, Chambert 1984, Amoros \& Chessel 1985, Jacquet-Labrot 1986, Carrel \& Juget 1987).

Bien qu'aucune étude n'ait été auparavant réalisée sur les bras morts précités de la Garonne, leurs potentialités écologiques ont été pressenties et des aménagements ont été réalisés. En particulier certains bras morts ont été recreusés pour retarder leur "vieillissement » (diminution des communications avec le fleuve, envasement, prolifération végétale), phénomène accéléré par les abaissements du litconsécutifs aux extractions de granulat (Beaudelin 1987).

Le présent travail, basé sur trois années d'observations (1988 à 1990), a pour double objectif :

- de connaître la composition de l'ichtyofaune (espèces de poissons, abondance relative et stades de développement) ;

- d'analyser les relations entre l'ichytyofaune et les caractéristiques des habitats.

A terme, cette étude vise à établir les bases d'une typologie fonctionnelle de l'ensemble des bras morts de la Garonne et à définir leur rôle dans le déroulement des processus biologiques intéressant les poissons.

\section{Matériel et méthodes}

\subsection{Description des sites étudiés}

Suite à une étude préliminaire menée sur six bras morts de la Garonne (Belaud et al. 1990), trois d'entre eux ont été jugés propices à des études plus longues, d'autant plus qu'ils sont restés en eau enconditions de sécheresses estivales en 1989 et 1990. Ces trois bras morts sont groupés dans le département de la Haute-Garonne en aval de Toulouse et sont répartis sur $6 \mathrm{~km}$ de fleuve. Ces sites retenus, d'amont vers l'aval, sont localisés dans la figure 1. Ils sont désignés par le lieu-dit ou l'agglomération la plus proche :

- Ramier de Bigorre (Seilh), désigné par l'abréviation « RB » : station 1 (amont) et 2 (aval),

- Port-Vieux (St. Caprais) ("PV ») : station 3 (amont) et 4 (aval),

- Grenade-Fontaine (Grenade) ("GF ») : station 5 (amont) et 6 (aval).

Les bras morts RB (PK : 693) et PV (PK : 697), recreusés artificiellement en 1986 et 1987 , respectivement, sont des exemples de réaménagements d'anciens bras morts naturels. Leur nouvelle forme rectiligne et leur fond relativement régulier tranchent avec leur morphologie d'origine. Le bras mort RB a une communication avec le fleuve à l'aval. A l'amont, une prise d'eau artificielle sur la Garonne relie celle-ci au bras par un canal étroit $(2 \mathrm{~m})$. Le bras mort PV a uniquement une communication à l'aval. Sa particularité est d'être séparé en deux parties par une buse de $100 \mathrm{~cm}$ de diamètre et sur une longueur d'environ $3 \mathrm{~m}$. Il n'a pas de communication avec la Garonne en amont. Ces deux bras morts récemment recreusés, sont relativement peu profonds (respectivement $1.2 \mathrm{~m}$ et $1.7 \mathrm{~m}$ en moyenne et pour un débit de la Garonne de $170 \mathrm{~m}^{3} / \mathrm{s}$ ), peu larges (respectivement $9.2 \mathrm{~m}$ et $15 \mathrm{~m}$ en moyenne) et à substrat grossier (graviers/galets). Le bras mort RB est plus long $(725 \mathrm{~m})$ que le bras mort PV $(364 \mathrm{~m})$.

Le bras mort GF (PK : 698) résulte d'une divagation de la Garonne et a conservé l'aspect naturel. Sa morphologie est asymétrique, la berge gauche correspondant à une petite falaise alors que la rive droite est pratiquement plane (zone inondable en période de crue). Il est peu profond (moyenne de $1.5 \mathrm{~m}$ ) mais à granulométrie fine (vase/sable) et large ( $32 \mathrm{~m}$ en moyenne), avec une longueur de $437 \mathrm{~m}$. 

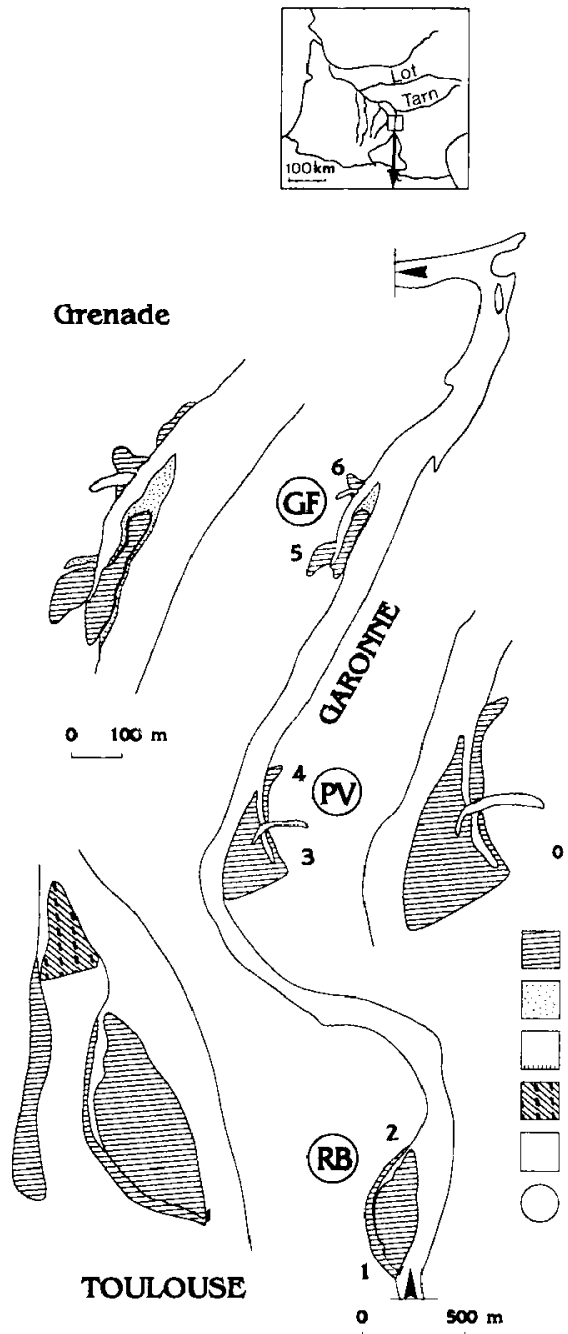

$\mathrm{N}$

St. Caprais

\section{Riplsylve}

Phalaridae

Saulale jeune

Cultures et peuplerales

Lande herbacee avec formations arbustlves

Bras mort

Fig. 1. Schéma de trois bras morts étudiés et emplacement des stations de prélévement (signalées par un chiffre).

Fig. 1. View of three ancient arms of the river and position of sampling stations (marked by a number). 


\subsection{Méthodes de pêche}

Douze campagnes d'observation ont été régulièrement assurées de février 1988 à décembre 1990 dans chacun des trois bras morts. Après divers essais, deux techniques de pêche ont été retenues : la pêche électrique et la pêche aux filets (Belaud et al. 1990). Ces deux techniques de pêche ont été mises en action en phases diurnes.

Les pêches électriques ont été pratiquées selon les techniques classiques (Vibert 1968, Lamarque et al. 1975), à l'aide d'un générateur de courant continu « Electro Pullmann » réglé entre 400 et 450 volts. Le courant a été établi entre une cathode fixe constituée d'un grillage immergé de surface adaptée et une anode mobile (anneau de cuivre de $35 \mathrm{~cm}$ de diamètre) attirant les poissons. Le courant délivré varie entre 3 et 4 ampères, pour une eau de température comprise entre 5,3 et $22.7^{\circ} \mathrm{C}$ et de conductivité électrique corrigée à $25^{\circ} \mathrm{C}$ comprise entre 220 et $340 \mu \mathrm{S} / \mathrm{cm}$. L'électrode a été manœuvrée selon le protocole de l'échantillonnage ponctuel d'abondance (E.P.A.) (Nelva et al. 1979). La méthode consiste à sonder des points répartis de manière aléatoire dans chaque bras mort. Le nombre de points pour chaque campagne de pêche et pour chaque bras mort varie entre 40 et 75 . En arrivant vers le point de pêche, l'opérateur effectue un mouvement de l'anode d'abord latéral puis vertical. Les poissons immobilisés sont immédiatement capturés pour procéder à l'identification et aux mensurations puis libérés sur place. Les résultats de captures sont exprimés en prise par unité d'effort (P.U.E.), l'unité d'effort étant définie comme une action d'échantillonnage ponctuel.

Parallèlement à la pêche, à chaque point de prêlèvement, les caractéristiques d'habitat ont été notées, selon une codification semi-quantitative portant sur la pente des berges, la profondeur, le substrat, la végétation aquatique, les abris divers et la ripisylve.

Les pêches aux filets ont été réalisées en utilisant une batterie de filets monofilaments expérimentaux de $15 \mathrm{~m}$ de long et $1.5 \mathrm{~m}$ de hauteur, avec des mailles de $10,17,21,27,34,44$ et $56 \mathrm{~mm}$. Ces filets couvrant toute la hauteur d'eau, ont été laissés en action pendant 30 minutes en divers endroits de chaque bras mort. Les résultats de captures sont également exprimés en prise par unité d'effort (P.U.E.), définie comme 30 minutes de pêche de l'ensemble de filets.
Les espèces de poissons ont été déterminées selon la clé de Spillmann (1961) et de Koblickaja (1981). La longueur totale a été mesurée à $1 \mathrm{~mm}$ près, et les poissons ont été pesés à $0.5 \mathrm{~g}$ près.

\subsection{Traitement numérique et statistique}

\subsubsection{Indices écologiques}

L'abondance spécifique est calculée en moyenne sur l'ensemble des 12 campagnes de prélèvements effectuées dans un bras mort donné. Elle est quantifiée d'une part, à partir des effectifs (abondance numérique) exprimée en nombre d'individus capturés par P.U.E., et d'autre part, à partir des biomasses (abondance pondérale) exprimée en grammes par P.U.E.

Les ressemblances des peuplements piscicoles de bras morts différents ont été évaluées par l'indice de Sorensen (So) pour la similitude qualitative (Brower \& Zar 1977, Legendre \& Legendre 1979, Magurran 1988) et par l'indice de Czekanovski (Cz) pour Ia similitude quantitative (Legendre \& Legendre 1979, Ludwig \& Reynolds 1988) :

So $=2 c /\left(S_{\mathrm{j}}+S_{\mathrm{j}}\right)$, avec $\mathrm{c}=$ nombre d'espèces communes aux deux bras morts comparés, $S_{\mathbf{i}}$ et $S_{j}$ $=$ nombre d'epèces des bras morts $\mathrm{i}$ et $\mathrm{j}$;

$\mathrm{Cz}=2 \mathrm{Nmn} /\left(\mathrm{N}_{\mathrm{i}}+\mathrm{N}_{\mathrm{j}}\right)$, avec $\mathrm{Nmn}=$ somme des abondances les plus faibles, pour chaque espèce, dans la paire de bras morts comparés i et $j, N_{j}$ et $\mathrm{N}_{\mathrm{j}}=$ somme des abondances spécifiques dans les bras morts $i$ et $j$.

La constance de l'espèce (C) est le pourcentage de prélèvements dans lesquels l'espèce est présente. Cet indice, qui se calcule sur l'ensemble des prélèvements réalisés dans un site donné, permet de distinguer trois classes (Bouvet et al. 1982 ; Gascuel 1985 ; Belaud et al. 1990): (1) espèces constantes $(\mathrm{C}>50 \%) ;(2)$ espèces accessoires $(25 \% \leqslant \mathrm{C} \leqslant$ $50 \%)$; (3) espèces accidentelles $(C<25 \%)$.

\subsubsection{Structure en groupes de classes de taille}

Les premières observations avaient suggéré d'anaiyser chaque peuplement ponctuel non seulement en tenant compte des espèces mais, aussi, au sein de chaque espèce, en distinguant les principaux groupes de classes de taille : juvéniles, immatures et adultes.

Après l'extraction des données des E.P.A., un tableau $\mathrm{N}$ a été construit, comportant I lignes (relevés) 
et $\mathrm{J}$ colonnes (classes de taille d'une espèce) où on trouve, à la ligne i et la colonne $\mathrm{j}$, l'effectif $\mathrm{N}_{\mathrm{ij}} \mathrm{d}$ 'individus de taille $j$ dans le relevé $i$. Un relevé définit un ensemble d'E.P.A. correspondant à un bras mort et une date donnée. Ce tableau a été ensuite traité par une analyse factorielle des correspondances (A.F.C.) (Badia \& Do Chi 1976, Do Chi 1978, Benzécri \& coll. 1982, Diday et al. 1982, Nelva 1989).

Le résultat de calculs de l'A.F.C. fait apparaître respectivement une liste des valeurs propres et un tableau de coordonnées des colonnes (classes de taille). Le graphe des valeurs propres a ensuite été tracé pour savoir combien de facteurs d'analyse parmi l'ensemble doivent être pris en considération. De même, les coordonnées factorielles des classes de taille ont été illustrées synthétiquement, pour chaque espèce retenue, sous la forme de cartes de coordonnées factorielles.

\subsubsection{Relations espèces-milieu}

L'étude quantitative de la répartition spatiale des espèces de poissons en fonction des caractéristiques du milieu, est souvent employée avec succès sur des variables qualitatives (Keast 1978a, Moreau \& Legendre 1979, Nelva 1985, Copp 1987). La mise en œuvre de cette étude dans les bras morts de la Garonne, a nécessité une adaptation méthodologique préalable. Cette adaptation a été réalisée à deux niveaux : celui de l'identification des principales caractéristiques de milieu d'une part, et celui du choix de l'analyse des données recueillies, d'autre part.

Après une étude préliminaire effectuée sur les six bras morts de la Garonne (Belaud et al. 1990), six caractéristiques du milieu ont été retenues ; elles ont été regroupées en modalités adaptées à la gamme rencontrée dans les trois milieux étudiés :

I. Situation $\mathrm{du}$ point d'échantillonnage (2 modalités) :
1. rive à pente faible ;
2. rive à pente moyenne/forte.

II. Profondeur de l'eau (4 modalités) :

3. inférieure à $0.5 \mathrm{~m}$;

4. comprise entre 0.5 et $1 \mathrm{~m}$;

5.1 à $1.5 \mathrm{~m}$;

6. supérieure à $1.5 \mathrm{~m}$.

III. Nature du substrat ( 3 modalités) : 7. limon-vase ;
8. vase-graviers ;

9. gravier-galets.

IV. Abris (3 modalités) :

10. absence ;

11. abris ligneux (branches, racines) présents ;

12. abris ligneux dominants.

V. Végétation aquatique ( 4 modalités) :

13. absence ;

14. type phragmites (semi-immergée) ;

15. type renoncules (totalement immergée) ;

16. type nymphea (flottante).

VI. Couvert ripisylve (2 modalités) :

17. absence ;

18. présence.

$\mathrm{Si}$, dans chaque modalité, on répartit sur l'ensemble des E.P.A., le nombre de poissons pour chacune des espèces rencontrées, on obtient alors un tableau de contingence espèces $x$ modalités.

La suite du traitement consiste en des analyses statistiques permettant d'associer, à l'organisation du milieu, une structure d'assemblages des espèces.

Deux types d'analyses multidimensionnelles ont été utilisées : l'Analyse Factorielle des Correspondances (A.F.C.) décrite par Lébart et al. (1979), Benzécri (1984), Foucart (1985), Digby \& Kempton (1987), Escofier \& Pagès (1988) en considérant les remarques de Chessel et al. (1982), Chessel \& Débouzie (1983) et la Classification Automatique par la méthode des nuées dynamique qui sont exposée principalement par Clifford \& Stephenson (1975), Legendre \& Legendre (1979), Roux (1985), Celeux (1989), Jambu (1989).

L'A.F.C. a été choisie en raison de son avantage de traiter un tableau de contingence et de permettre en particulier la mise en évidence des corrélations non linéaires. Elle répartit dans un espace multidimensionnel deux ensembles de points : d'une part, les espèces divisées en groupes de classes de taille dont chacune constitue une des lignes du tableau, et correspond donc à un individu statistique, et d'autre part, les modalités de chaque variable de milieu introduite dans le tableau initial dont chacune constitue une des colonnes du tableau. La position de ces points est déterminée par leurs coordonnées sur les axes qui définissent l'espace (axes factoriels). On a ajouté à ces deux ensembles une troisième série de variables supplémentaires : les trois bras morts séparés en deux modalités correspondant respectivement 
aux zones amont et aval. Ces points viendront alors se placer dans l'espace, sans en modifier la construction. Leur positionnement par rapport à ces axes constitue une aide à l'interprétation.

Après l'ensemble des calculs de l'A.F.C., on représentera graphiquement dans les différents plans factoriels : d'une part, les individus statistiques (especes en différents groupes de taille), et d'autre part, les modalités des variables de milieu. La représentation des espèces peut également être obtenue par rapport aux variables supplémentaires (bras morts). Chacun de ces trois bras morts a été divisé en deux parties : amont (AM) et aval (AV). Ces différentes représentations mettent en évidence les groupements entre espèces et permettent d'analyser ces groupements en relation avec les caractéristiques du milieu et avec le bras mort concerné.

Le classement automatique des individus statist $i-$ ques permet de regrouper les points du nuage, en différentes classes, en fonction de leur proximité dans l'espace vectoriel considéré. Employée à l'issue de l'A.F.C., cette méthode permet de formaliser les regroupements observés dans différents plans factoriels ; l'espace vectoriel pris en compte est donc celui formé par les premiers axes factoriels considérés. Le dendrogramme construit utilise l'algorithme d'une classification ascendante hiérarchique où l'abondance a été calculée sur la distance du khideux. Le critère d'agrégation choisi est celui du moment d'ordre deux qui a une forte tendance à former des groupes. Ce dendrogramme permet de juger de la proximité des différentes classes mises en évidence et éventuellement de les regrouper en superclasses, ou de les subdiviser en sous-classes.

\subsubsection{Relations espèces-temps}

Dans le but de mettre en évidence une typologie des espèces rendant compte des variations temporelles, l'A.F.C. et la classification automatique par la méthode des nuées dynamiques ont été également utilisées.

Le tableau des données construit, s'attache aussi à la description d'un tableau de $\mathrm{n}$ lignes (individus statistiques) caractérisés par $\mathrm{p}$ variables (dates de pêches). On considère chacune des espèces regroupées en classes de taille comme un individu ; sa densité numérique dans les différentes dates de pêches est alors assimilée à la réalisation d'une variable. Chaque date de prélèvements était une saison (Hiver $\mathrm{H}$, Printemps $\mathbf{P}$, Eté $\mathbf{E}$ ou Automne A) à une année donnée. Ce tableau définit ainsi un nuage de n points, dans un espace vectoriel de dimension $p$; il constitue un tableau de contingence. La suite du traitement se fait comme expliqué précédemment.

Cette méthode d'analyse factorielle consiste à rechercher la meilleure représentation possible de ce nuage de points, c'est-à-dire celle absorbant le maximum d'inertie, dans un espace de dimension plus faible que l'espace initial. La représentation des individus statistiques (espèces à différents groupes de taille) dans les différents plans factoriels permet alors d'étudier leurs regroupements. Ces regroupements sont ensuite formalisés par la classification automatique débouchant sur : d'une part, la construction du dendrogramme qui visualise les distances entre classes ou sous-classes, et d'autre part, la description de chacune des classes par les variables (dates de prélèvements) les plus caractéristiques.

\section{Résultats}

\subsection{Composition et abondances spécifiques}

Dans l'ensemble des bras morts étudiés et les deux techniques de pêche confondues, un total de 24 espèces dont 20 à Ramier de Bigorre (RB), 23 à PortVieux (PV) et 23 à Grenade-Fontaine (GF), a été recensé lors des 12 campagnes de pêches effectuées de février 1988 à décembre 1990 (Tableau 1). Les résultats de pêche obtenus par les E.P.A. et par les filets sont présentés dans les Tableaux 2 et 3 , respectivement.

Sur l'ensemble des pêches, quatre espèces observées par E.P.A. ne sont pas capturés dans les filets. Il s'agit de la soiffe (Chondrostoma toxostoma), la gambusie (Gambusia affinis), l'anguille (Anguilla anguilla) et la lamproie de planer (Lampetra planeri). A l'inverse, aucune espece observée aux filets n'échappe aux E.P.A. La majorité des poissonscontrôlés par les E.P.A. sont de petite taille ( $\mathrm{L} t<$ $150 \mathrm{~mm}$ ). Les filets complètent les captures par des poissons adultes de grande taille.

La forte similitude qualitative entre $\mathrm{RB}$ et $\mathrm{PV}$ (So $=0.93$ ) et entre PV et $G F(S o=0.96$ ) révèle les affinités spécifiques du peuplement piscicole fréquentant ces trois bras morts. Parmi les huit familles inventoriées, les cyprinidés dominent largement en nombre d'espèces ( $58,3 \%$ du peuplement global). Les différences spécifiques intersites ne portent que sur des espèces rares $(C<10 \%$ ) (soiffe, lamproie de planer et grémille). 
Tableau 1. Liste des espèces de poissons recensées dans les trois bras morts de la Garonne.

Table 1. List of fish species recorded in three ancient arms of the Garonne.

\begin{tabular}{|c|c|c|c|c|c|c|}
\hline FAMILLE & ESPECES & NOM & CODE & RB & PV & GF \\
\hline CYPRINIDAE & 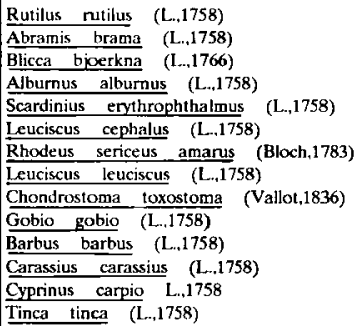 & $\begin{array}{l}\text { Gardion } \\
\text { Brème } \\
\text { Brème bordelière } \\
\text { Ablette } \\
\text { Rotengle } \\
\text { Chevaine } \\
\text { Bouvière } \\
\text { Vandoise } \\
\text { Soiffe } \\
\text { Goujon } \\
\text { Barbeau } \\
\text { Carassin } \\
\text { Carpe commune } \\
\text { Tanche }\end{array}$ & $\begin{array}{l}\text { GAR } \\
\text { BRE } \\
\text { BRB } \\
\text { ABL } \\
\text { ROT } \\
\text { CHE } \\
\text { BOU } \\
\text { VAN } \\
\text { TOX } \\
\text { GOU } \\
\text { BAF } \\
\text { CAS } \\
\text { CCO } \\
\text { TAN }\end{array}$ & $\begin{array}{l}\cdot \\
: \\
: \\
: \\
: \\
:\end{array}$ & $\begin{array}{l}: \\
* \\
* \\
* \\
* \\
*\end{array}$ & $\begin{array}{l}: \\
: \\
: \\
: \\
: \\
: \\
: \\
* \\
* \\
* \\
*\end{array}$ \\
\hline POECILIIDAE & Gambusia affinis (L.,1758) & Gambusie & GAM & & - & $*$ \\
\hline ICTALURIDAE & Ictalurus melas (Rafinesque,1820) & Poisson-chat & $\mathrm{PCH}$ & - & $*$ & $*$ \\
\hline ANGUILLIDAE & Anguilla anguilla (L..1758) & Anguille & ANG & - & $*$ & $\bullet$ \\
\hline PETROMYZONIDAE & Lampetra_planeri (Bloch,1786) & Lamproie de planer & LPP & & * & \\
\hline PERCIDAE & 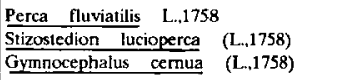 & $\begin{array}{l}\text { Perche } \\
\text { Sandre } \\
\text { Grémille }\end{array}$ & $\begin{array}{l}\text { PER } \\
\text { SAN } \\
\text { GRE }\end{array}$ & . & * & $\stackrel{\bullet}{*}$ \\
\hline CENTRARCHIDAE & $\begin{array}{l}\text { Micropterus salmoides }(\text { L.,1758) } \\
\text { Lepomis gibbosus }(\text { L., 1758) }\end{array}$ & $\begin{array}{l}\text { Black-bass } \\
\text { Perche-soleil }\end{array}$ & $\begin{array}{l}\text { BBG } \\
\text { PES }\end{array}$ & - & 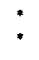 & $\ddot{*}$ \\
\hline ESOCIDAE & Esox lucius L.,1758 & Brochet & BRO & * & • & * \\
\hline
\end{tabular}

Les abondances numériques et pondérales par P.U.E. sont globalement plus fluctuantes aux bras morts RB et PV qu'à GF (le coefficient de variation $C V$ étant plus fort pour ces deux premiers bras morts) (Tableaux 2 et 3 ). Toutefois, la forte similitude quantitative évaluée par l'indice de Czekanovski ( $\mathrm{Cz}=0.74$ entre $\mathrm{RB}$ et $\mathrm{PV}, \mathrm{Cz}=0.73$ entre $\mathrm{RB}$ et $\mathrm{GF}, \mathrm{Cz}=0.77$ entre $\mathrm{PV}$ et $\mathrm{GF}$ ), confirme l'homogénéité numérique des communautés piscicoles de ces trois bras morts. Il en est de même pour l'abondance pondérale entre RB et $\mathrm{PV}(\mathrm{Cz}=0.77)$. En revanche, la similitude pondérale est relativement faible entre le peuplement du bras mort RB et GF $(\mathrm{Cz}=0.55)$, ainsi qu'entre $\mathrm{PV}$ et $\mathrm{GF}(\mathrm{Cz}=0.56)$. Ces différences résultent d'une abondance d'adultes de grandes espèces au bras mort GF.
Sur les 24 espèces observées, il existe onze espèces constantes $(\mathrm{C}>50 \%)$ dans les bras morts mais dont les effectifs et les biomasses sont différents (Tableaux 2 et 3). Elles appartiennent aux quatre familles : cyprinidés (gardon, brème, rotengle, bouvière, carassin, carpe, tanche) ; ictaluridés (poissonchat) ; percidés (perche) ; centrarchidés (black-bass et perche-soleil).

Le peuplement du bras mort RB est numériquement dominé par le gardon tant dans les E.P.A. que lors de pêche aux filets ( $22.9 \%$ et $31.9 \%$ respectivement), suivi par la brème et la bouvière si on prend les données des E.P.A. et par la brème et le poissonchat si on prend les données des pêches aux filets. Le poisson-chat domine en poids dans les E.P.A. 


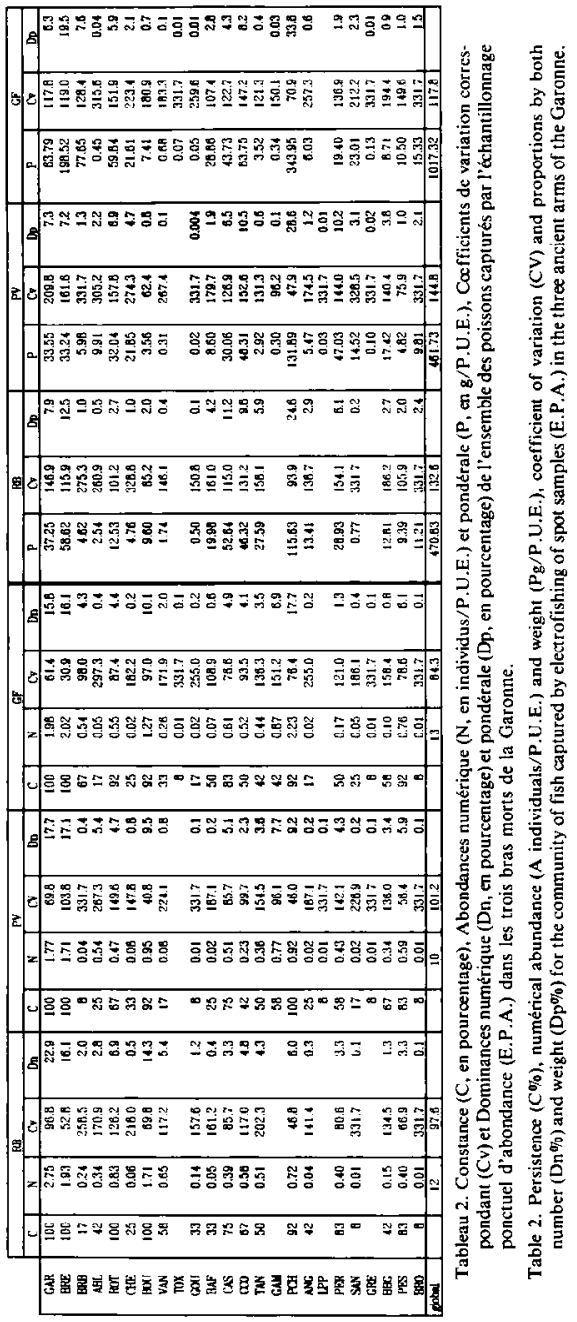

\begin{tabular}{|c|c|c|c|c|}
\hline & S? & $2=38$ & 产 & 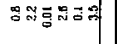 \\
\hline & 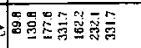 & 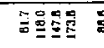 & 8 & 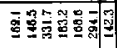 \\
\hline & 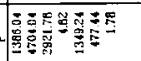 & 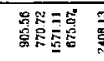 & $\frac{9}{9}$ & -3 \\
\hline & 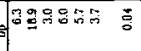 & $=\infty+\infty$ & $\underline{2}$ & $\sqrt{n+5}$ \\
\hline & 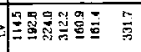 & 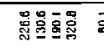 & $\overline{\mathbf{a}}$ & 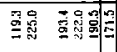 \\
\hline & 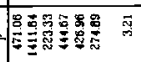 & 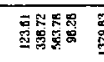 & 㩊 & 羿: \\
\hline & 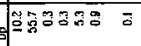 & $5:$ & 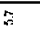 & 99 \\
\hline & 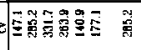 & 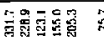 & 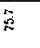 & 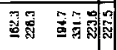 \\
\hline & 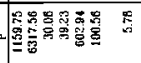 & 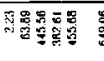 & 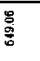 & 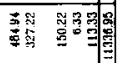 \\
\hline & 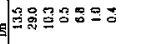 & 4050 & $\stackrel{g}{g}$ & 909098 \\
\hline & 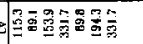 & 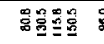 & 8 & 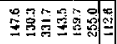 \\
\hline & 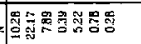 & 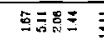 & $\equiv$ & 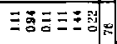 \\
\hline & $28 x=5 x=$ & 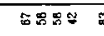 & $\mathrm{g}$ & 98080 \\
\hline & 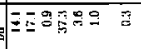 & 5973 & 5 & 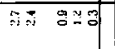 \\
\hline & 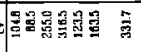 & 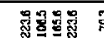 & $g$ & 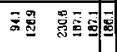 \\
\hline & 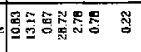 & 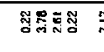 & $\frac{\pi}{2}$ & 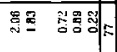 \\
\hline & 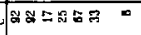 & $=585:$ & $\bar{y}$ & 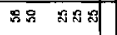 \\
\hline & 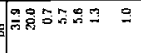 & $938 \div$ & $\overline{5}$ & 뭄 95 \\
\hline & 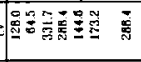 & 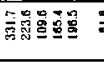 & $\ddot{9}$ & 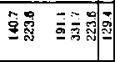 \\
\hline & 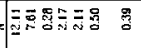 & $\overline{0}$ & 票 & 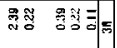 \\
\hline & $38 \infty \approx 7 \infty$ & 7 & 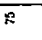 & $\because 1$ \\
\hline & 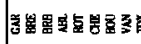 & & & \\
\hline
\end{tabular}


$(24.6 \%)$ et la brème dans les captures aux filets $(55.7 \%$ ). Les carnassiers représentés par la perche, le sandre, le black-bass, la perche-soleil et le brochet n'ont constitué que $8.1 \%$ du nombre d'individus et $13.4 \%$ du poids total des poissons observés lors des E.P.A. Ils sont également moins nombreux dans les captures aux filets où ils n'ont représenté que $8.8 \%$ en effectif et $9.6 \%$ en biomasse.

Comme le bras mort précédent, le peuplement du bras mort PV est aussi dominé en nombre par le gardon et la brème ( $17.7 \%$ et $17.1 \%$ respectivement) dans les E.P.A. et l'ablette $(37.3 \%)$ dans les filets. A l'inverse, le poisson-chat et la carpe occupent pondéralement une place importante (respectivement $28.6 \%$ et $10.5 \%$ ) dans les E.P.A. et la brème et le gardon (respectivement $55.7 \%$ et $10.2 \%$ ) dans les filets. Les carnassiers pêchés par les E.P.A. et par les filets ne sont représentés essentieliement que par quatre espèces (perche, sandre, black-bass et perche-soleil) constituant globalement $13.8 \%$ du nombre d'individus et $18.1 \%$ du poids total des poissons pour les E.P.A. et $7.2 \%$ en effectif et $19.4 \%$ en biomasse pour les filets.

Sur l'ensemble des captures du bras mort GF, le gardon et la brème sont également les mieux représentés en nombre dans les E.P.A. (31.9\%) et dans les filets $(42.5 \%)$, suivies par la bouvière $(10.1 \%)$ lors des E.P.A. et la brème bordelière $(10.3 \%)$ lors des pêches aux filets. Parmi ces quatre cyprinidés, la brème est pondéralement la seule espèce dominante tant dans les E.P.A. $(19.5 \%$ ) que dans les filets $(24.9 \%)$, vient ensuite la brème bordelière seulement pour les pêches aux filets $(15.4 \%)$. Toutefois, le poisson-chat occupe régulièrement une place aussi importante tant numérique $(17.7 \%$ dans les E.P.A. et $18.5 \%$ dans les filets) que pondérale ( $33.8 \%$ dans les E.P.A. et $12.7 \%$ dans les filets). Les carnassiers représentés en grande partie par la perche, le sandre, le black-bass et la perche-soleil, sont plus ou moins équilibrés numériquement et pondéralement, avec un léger avantage en nombre de la perche-soleil. La grémille et le brochet sont les deux espèces carnassières également présentes dans ce bras mort, mais avec un très faible effectif.

\subsection{Structure en groupes de classes de taille}

Pour aborder cette étude, les contraintes d'analyse ont imposé l'élimination des espèces peu représentées. Les onze espèces constantes ( $C>50 \%$ ) extraites des 36 pêches (1601 E.P.A.) effectuées dans l'ensemble des trois bras morts, ont été retenues. Il s'agit des gardons (GAR), brèmes (BRE), rotengles (ROT), bouvières (BOU), carassins (CAS), carpes (CCO), tanches (TAN), poissons-chat ( $\mathrm{PCH})$. perches-soleil (PES), perches (PER) et black-bass (BBG).

Les résultats de l'A.F.C. de chaque espèce sont représentés synthétiquement sous forme des cartes de coordonnées factorielles dans les figures $2 a, 2 b$, $2 c$ et $2 \mathrm{~d}$. Le dépouillement simultané des 5 premiers facteurs met en évidence, pour chaque espèce, les ruptures dans les coordonnées factorielles des classes de taille. Ces ruptures confirment les groupements par classes de taille. Elles ne sont, en effet, bien distinctes que sur certains premiers facteurs examinés sur le graphe des valeurs propres (illustrés ici par les flèches).

Les différents groupes de classes de taille disjoints correspondent significativement aux zones successivement occupées au cours du développement de chacune des onze espèces. Ces groupes respectifs et leurs âges correspondants sont résumés dans le Tableau 4. Ils font partie par la suite de l'analyse des relations espèces-milieu-temps.

\subsection{Organisation spatiale}

Le résultat de l'A.F.C. portant sur 28 groupes de classes de taille appartenant à 11 espèces de poissons et sur 18 modalités des 6 variables de milieu, indique que l'essentiel de l'information est concentré sur les deux premiers facteurs (plan F1 $\times$ F2) rendant compte de $70.3 \%$ de l'inertie totale du nuage des points : $52.6 \%$ pour le premier et $17.7 \%$ pour le second (Fig. 3). Le troisième facteur examiné (9.3\% d'inertie expliquée) apporte très peu de renseignements par rapport aux deux premiers.

Trois groupements sont ainsi bien délimités sur le plan des axes F1 et F2. Ces trois groupements sont confirmés par la classification automatique des espèces à différents groupes de taille (Fig. 4) et des modalités de variables du milieu (Fig. 5), réalisée sur les trois premiers facteurs de l'A.F.C.

Etablis à l'issues de l'A.F.C. et de la classification hiérarchique, ces trois groupes permettent de définir les systèmes d'axes adéquats, les espèces qu'ils rassemblent et les caractéristiques du biotope qu'ils associent, ainsi que les bras morts où ces associations sont les plus fréquentes. 


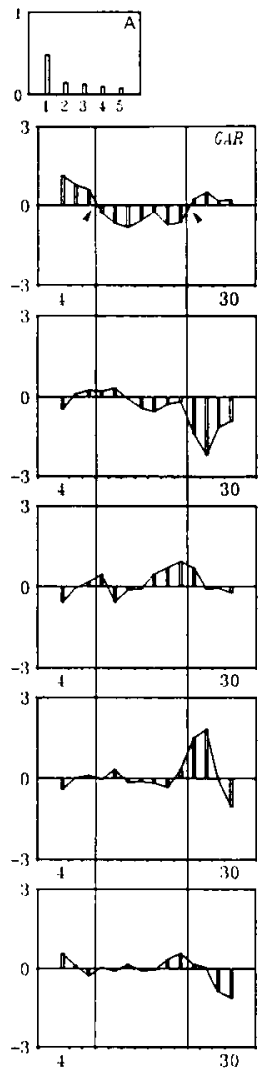

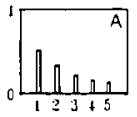
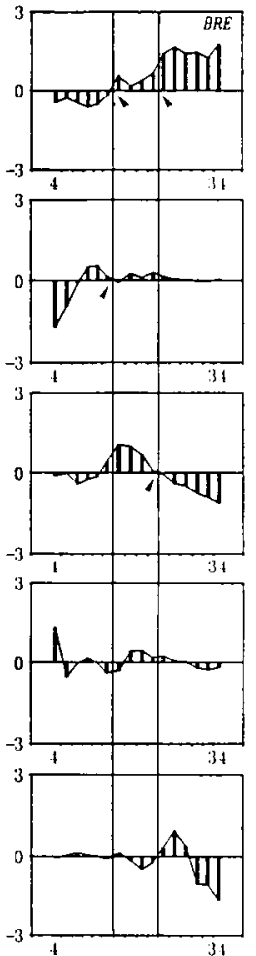
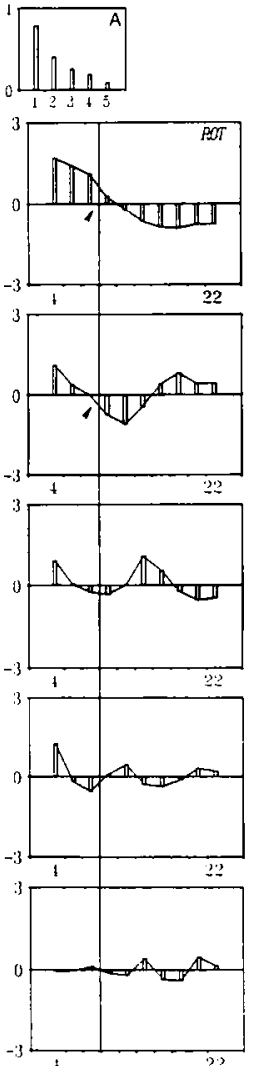

Fig. 2a. Représentation simultanée du graphe des valeurs propres (A) et des coordonnées factorielles des classes de taille pour le gardon, la brème et la rotengle sur les 5 premiers facteurs de l'A.F.C

Fig. 2a. Simultaneous graphical representation. of the eigenvalues (A) and factorial coordinates of size classes for roach, bream and rudd on the first five factors of A.F.C. (correspondence analysis).

Le groupe I est essentiellement construit sur le premier facteur ( $F 1)$ d'une part (corrélation positive) et sur le second facteur (F2) d'autre part (corrélation négative). Il est largement individualisé par les petites espèces (bouvière $\mathrm{BO} 1, \mathrm{BO} 2$ ) et les juvéniles des grandes espèces (gardon GA1, carassin CAl, carpe $\mathrm{CC} 1$, tanche $\mathrm{TN} 1$ ). Ces espèces dominantes s'associent également aux immatures des plus grandes espèces, principalement de carassin (CA2), tanche (TN2) et poisson-chat ( $\mathrm{PC} 2$ ) ; elles caractérisent 

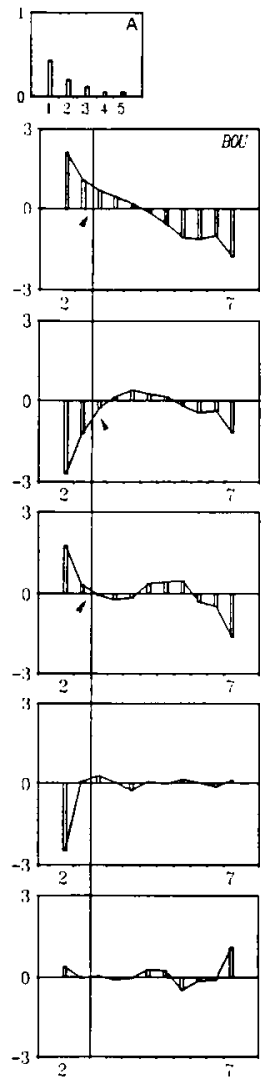
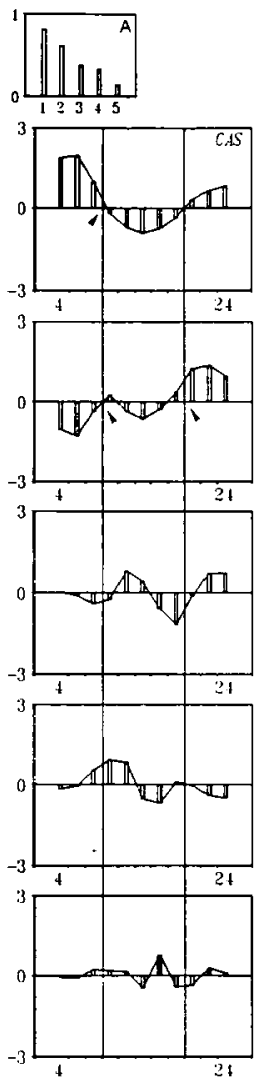
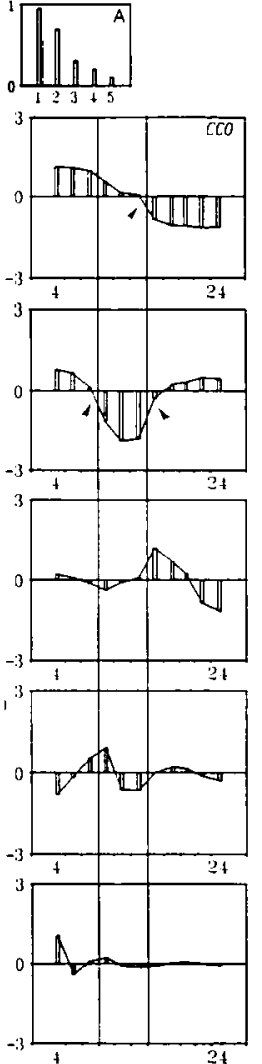

Fig. 2b. Représentation simultanée du graphe des valeurs propres (A) et des coordonnées factorielles des classes de taille pour la bouvière, le carassin et la carpe sur les 5 premiers facteurs de l'A.F.C

Fig. 2b. Simultaneous graphical representation of the eigenvalues (A) and factorial coordinates of size classes for bitterling, crucian carp and carp on the first five factors of A.F.C. (correspondence analysis).

pour l'essentiel les zones à pente faible (1) et peu profondes (3) où se trouvent les substrats à granulométrie limon-vase (7) et la végétation aquatique à prédominance de renoncules $(15)$ et de nymphea (16) auxquelles correspondent l'amont du bras mort GF (GFAM) et la partie plus aval du bras mort RB (RBAV).
Le groupe II met en évidence, sur la partie positive de l'axe 2 , l'influence de la végétation aquatique, notamment de type phragmites (14) et de la ripisylve (18) sur la répartition des petites espèces carnassières comme perche-soleil (PS1, PS2) et des juvéniles des plus grandes espèces carnassières comme perche (PE1) et black-bass (BB1). 

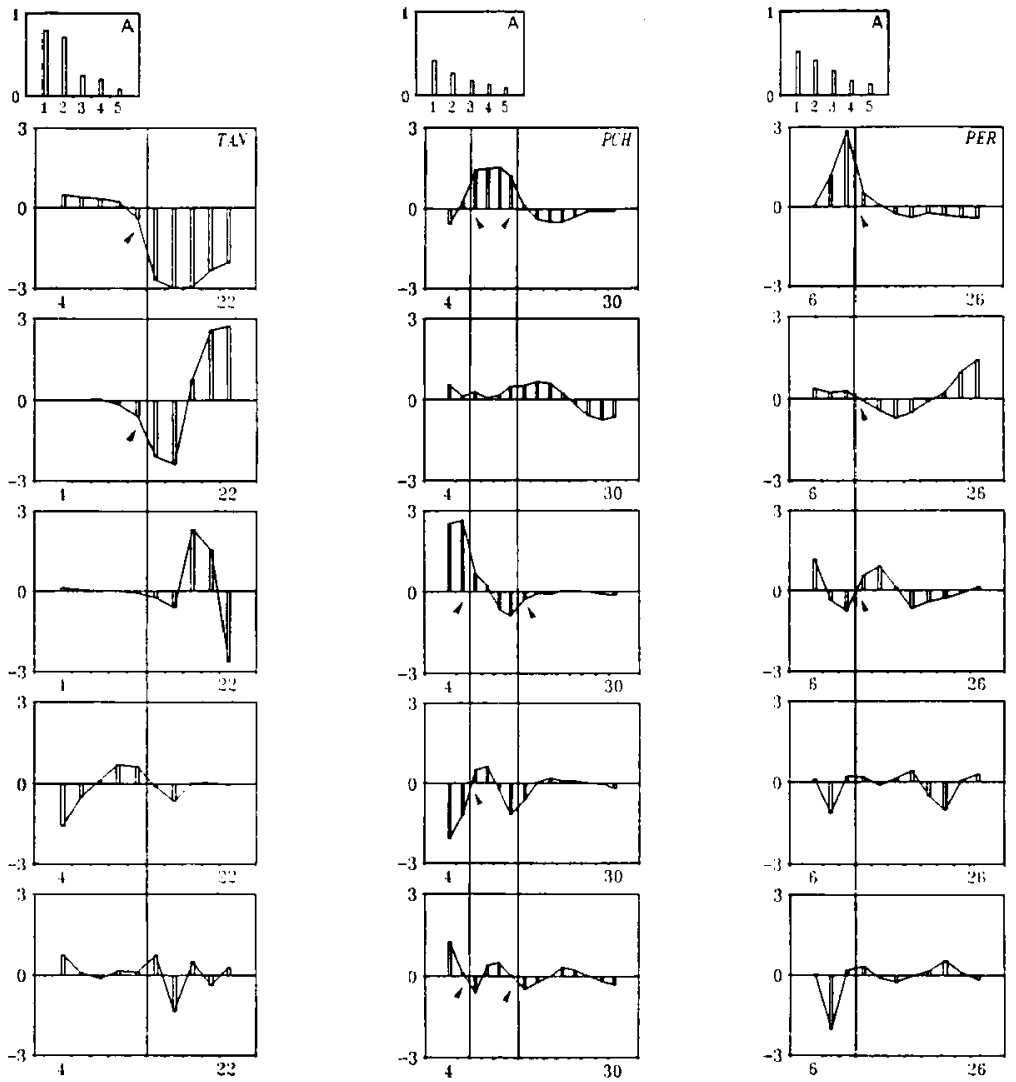

Fig. 2c. Représentation simultanée du graphe des valeurs propres (A) et des coordonnées factorielles des classes de taille pour la tanche, le poisson-chat et la perche sur les 5 premiers facteurs de l'A.F.C.

Fig. 2c. Simultaneous graphical representation of the eigenvalues $(A)$ and factorial coordinates of size classes for tench, catfish and perch on the first five factors of A.F.C. (correspondance analysis).

Le groupe III est bien défini sur l'axe 1 (corrélation négative). Il prend essentiellement en compte les modalités du biotope : rives à pente moyenne ou forte (2), avec profondeur supérieure à $1 \mathrm{~m}(5)$ en bordure des bancs de gravier-galets (9), hors de zones d'abris (10) auxquelles sont fortement associées les adultes de gardon (GA3), brème (BR3), carassin (CA3) et perche (PE2) représentatives des zones plus aval des bras morts PV (PVAV) et GF (GFAV). 

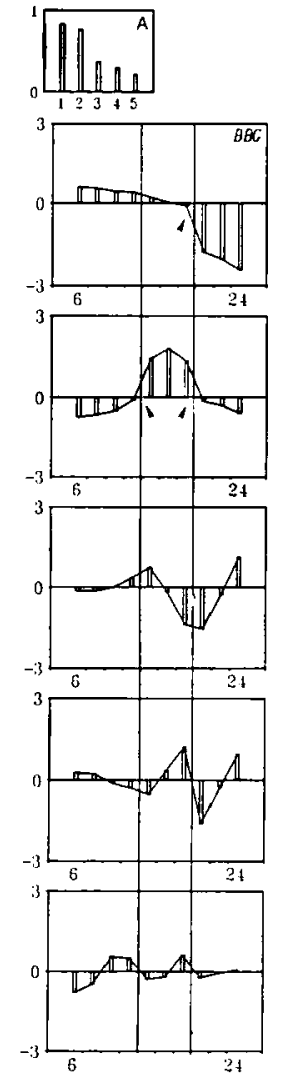
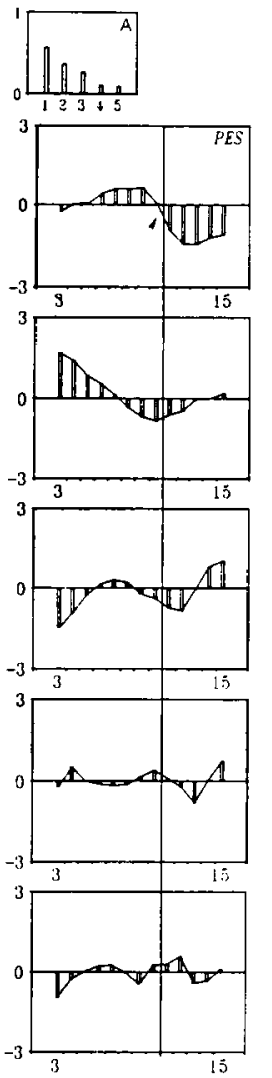

Fig. 2d. Représentation simultanée du graphe des valeurs propres (A) et des coordonnées factorielles des classes de taille pour le blackbass et la perche-soleil sur les 5 premiers facteurs de l'A.F.C.

Fig, 2d. Simultaneous graphical representation of the eigenvalues (A) and factorial coordinates of size classes for black bass and sunfish on the first five factors of A.F.C. (correspondence analysis).

\subsection{Variabilité saisonnière}

L'A.F.C. effectuée sur le tableau constitué des 28 groupes d'espèces et des 12 dates des campagnes de prélèvements, a montré que les plus fortes contributions étaient determinées sur les deux premiers axes (plan F1 $\times$ F2) permettant d'expliquer respectivement $24.7 \%$ et $23.4 \%$ de l'inertie du nuage, soit au total $48.1 \%$ (Fig. 6). La signification de cette structure est comparable à celle de l'analyse précédente. 
Tableau 4. Séparation des captures par espèce et par groupes de tailles correspondant aux différents stades de développement.

Table 4. Separation of catch by species and by size groups corresponding to the different development stages.

\begin{tabular}{|c|c|c|c|}
\hline Espéces & Code & $\begin{array}{c}\text { Groupes de LT } \\
(\mathrm{cm})\end{array}$ & $\begin{array}{c}\text { Groupes d'âge } \\
\text { (ans) }\end{array}$ \\
\hline Gardon & $\begin{array}{l}\mathrm{GA1} \\
\mathrm{GA} 2 \\
\mathrm{GA} 3\end{array}$ & $\begin{array}{c}4-8 \\
10-22 \\
24-30\end{array}$ & $\begin{array}{c}0+ \\
1 \dot{\mathrm{a}} 2 \\
2+\dot{\mathrm{a}} 4\end{array}$ \\
\hline Brème & $\begin{array}{l}\mathrm{BR} 1 \\
\mathrm{BR} 2 \\
\mathrm{BR} 3\end{array}$ & $\begin{array}{c}4-14 \\
16-22 \\
24-34\end{array}$ & $\begin{array}{c}0+\mathbf{a} 1 \\
1+\dot{\mathbf{a}} 2 \\
2+\dot{\mathbf{a}} 3+\end{array}$ \\
\hline Rotengle & $\begin{array}{l}\mathrm{RO1} \\
\mathrm{ROO}\end{array}$ & $\begin{array}{c}4-8 \\
10-22\end{array}$ & $\begin{array}{c}0+ \\
1: 2+\end{array}$ \\
\hline Bouvière & $\begin{array}{l}\mathrm{BO1} \\
\mathrm{BO2} \\
\end{array}$ & $\begin{array}{c}2-2.5 \\
3-7 \\
\end{array}$ & $\begin{array}{c}0+ \\
1-3 \\
\end{array}$ \\
\hline Carassin & $\begin{array}{l}\mathrm{CA} 1 \\
\mathrm{CA} 2 \\
\mathrm{CA} 3 \\
\end{array}$ & $\begin{array}{c}4-8 \\
10-18 \\
20-24\end{array}$ & $\begin{array}{c}0+ \\
1 \text { à } 2 \\
2+\dot{a} 3\end{array}$ \\
\hline Carpe & $\begin{array}{l}\mathrm{CC} 1 \\
\mathrm{CC} 2 \\
\mathrm{CC} 3\end{array}$ & $\begin{array}{c}4-8 \\
10-14 \\
16-24\end{array}$ & $\begin{array}{c}0+ \\
0+\dot{a} 1 \\
1+\dot{a} 2\end{array}$ \\
\hline Tanche & $\begin{array}{l}\mathrm{TN1} \\
\mathrm{TN2} \\
\end{array}$ & $\begin{array}{c}4-12 \\
14-22 \\
\end{array}$ & $\begin{array}{c}0+ \\
1 \dot{\mathrm{a}} 2 \\
\end{array}$ \\
\hline Poisson-chat & $\begin{array}{l}\mathrm{PC1} \\
\mathrm{PC2} \\
\mathrm{PC} 3\end{array}$ & $\begin{array}{c}4-6 \\
8-14 \\
16-30\end{array}$ & $\begin{array}{c}0+ \\
0+\text { à } 1+ \\
2 \dot{a} 4+\end{array}$ \\
\hline Perche & $\begin{array}{l}\mathrm{PE} 1 \\
\mathrm{PE} 2\end{array}$ & $\begin{array}{c}6-10 \\
12-26 \\
\end{array}$ & $\begin{array}{c}0+\dot{a} 1 \\
1+\text { a } 3+\end{array}$ \\
\hline Black-bass & $\begin{array}{l}\mathrm{BB} 1 \\
\mathrm{BB} 2 \\
\mathrm{BB} 3 \\
\end{array}$ & $\begin{array}{c}6-12 \\
14-18 \\
20-24\end{array}$ & $\begin{array}{l}0+\dot{a} 1 \\
1+\dot{a} 2 \\
2+\dot{a} 3 \\
\end{array}$ \\
\hline Perche-soleil & $\begin{array}{l}\text { PS1 } \\
\text { PS2 }\end{array}$ & $\begin{array}{c}3-10 \\
11-15\end{array}$ & $\begin{array}{c}0+\dot{a} 2 \\
2+\dot{a} 3+\end{array}$ \\
\hline
\end{tabular}

L'axe 1 est essentiellement déterminé, d'une part (partie positive de F1), par les observations en période de hautes eaux de printemps (P89, P90) où s'individualisent les adultes d'espèces de grande taille représentées par le gardon (GA3), la brème (BR3), la carpe $(\mathrm{CC} 3)$, le poisson-chat $(\mathrm{PC} 3)$, la perche (PE2) et le black-bass (BB3), et d'autre part (F1 négative), par les observations en période de basses eaux de l'été à l'automne 1989 (E89, A89) caractérisés par une dominance des petites espèces (bouvière $\mathrm{BO} 2$ et perche-soleil PS2), des juvéniles (rotengle $\mathrm{RO} 1$ et tanche TN1) et des immatures (carassin CA2 et poisson-chat $\mathrm{PC} 2$ ) de plus grandes especes.

L'axe 2 cst essenticllement identifić dans un sens par les observations en période de basses eaux estivales (E88, E90) auxquels sont principalement associés des juvéniles de plus grandes espèces (gardon GA1, carpe $\mathrm{CCl}$ et black-bass BB1). Dans le sens 


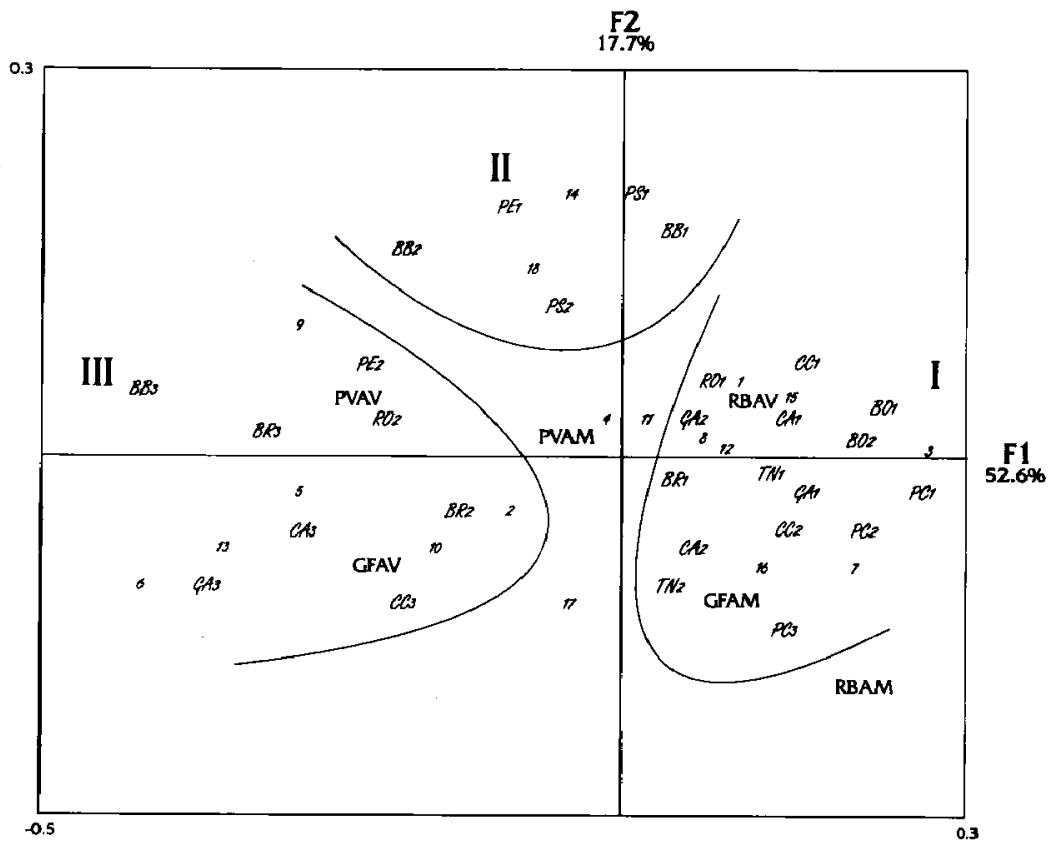

Fig. 3. Analyse factorielte des correspondances. Projection simultanée des 18 variables d'habitat et des 11 espèces subdivisèes en groupes de taille, dans le plan des deux premiers axes factoriels. Les six stations de trois bras morts projetés en éléments supplémentaires sont indiquées par une abréviation de 4 letures.

Fig. 3. Correspondence analysis. Simultaneous projection of 18 habitat variables and 11 species subdivided into size groups, arranged according to the first two major axes. The six sampling stations on the three ancient arms of the river are indicated by an abbreviation in four letters.

opposé, les observations d'hiver (H88, H90) sont caractérisés par une importance des cyprinidés immatures (gardon GA2, brème $\mathrm{BR} 2$ et rotengle $\mathrm{RO} 2$ ).

En terme d'analyse canonique, trois groupements apparaissent bien isolés dans le plan $F 1 \times F 2$; ils correspondent aussi aux trois groupes principaux établis par la classification hiérarchique à partir de l'espace des axes factoriels 1 à 3 (Fig. 7 et 8). Ces trois groupements se font essentiellement sur les différentes phases hydrologiques (crue et étiage) qui se succèdent dans l'année.
Le premier correspond aux observations effectuées en période de très basses eaux estivales 1989-1990 et qui durent jusqu'à l'automne. Ils sont caractérisés par une abondance exceptionnelle des petites espèces (bouvière et perche-soleil), ainsi que les juvéniles des plus grands cyprinidés.

Le second ne regroupe que les observations d'hiver 1988 et 1990 qui sont également caractérisés par les cyprinidés opportunistes (gardon, brème et rotengle), constamment présents dans les bras morts.

Le groupe III rassemble les observations effectuées en périodes de crues printanières qui sont 


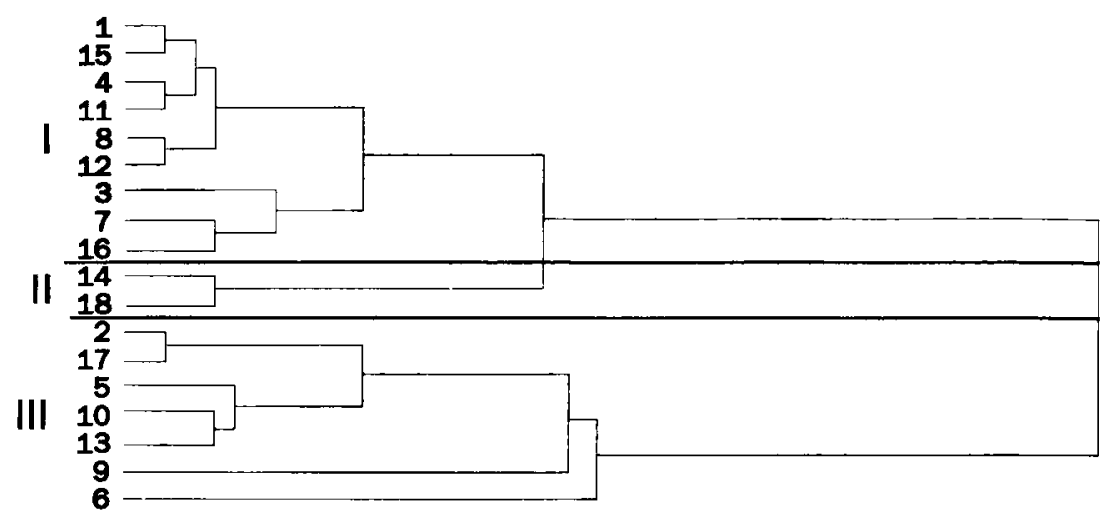

Fig. 4. Dendrogramme de la classification hiérarchique des 18 variables d'habitat sur les trois premiers facteurs de l'analyse factorielle des correspondances.

Fig. 4. Dendrogram for the hierarchical classification of 18 habitat variables on the first three factors from the correspondence analysis.

dominés par des poissons adulies appartenant aux cyprinidés (gardon, brème et carpe) et également aux poissons de fond (poisson-chat) et aux carnassiers (perche et black-bass).

\section{Discussion et conclusion}

Des deux méthodes de pêche mises en œuvre, l'échantillonnage ponctuel d'abondance (E.P.A.) par pêche électrique présente des avantages par rapport aux échantillonnages aux filets. Non seulement la composition spécifique inventoriée est plus élevée (Bengen 1988, Falta 1988, Belaud et al. 1990), mais encore les structures écologiques (structures des populations en classe de taille, relations interspécifiques, profils écologiques...) sont mieux établies. Le seul avantage de la péche aux filets est la prise en compte des individus de grande taille difficiles à surprendre par la pêche électrique. Les deux techniques complémentaires sont donc recommandées pour étudier ce type de milieux.

Les conditions physiques du milieu telles que la profondeur, la conductivité et la température de l'eau, influent sur l'efficacité de la pêche électrique (Vibert 1968, Cross 1976). La hauteur d'eau des bras morts étudiés $(<1.5 \mathrm{~m})$, ainsi que la conductivité électrique, entre 220 et $340 \mu \mathrm{S} / \mathrm{cm}$ et la température de l'eau, entre 5.3 et $22.7^{\circ} \mathrm{C}$ (Bengen et al. 1992), sont favorables à la pratique de la pêche électrique (Zalewski 1986). Les captures obtenues par E.P.A. constituent donc une base complète pour aborder la typologie ichtyenne des trois bras morts de la Garonne.

Les peuplements icht yques de ces trois bras morts présentent une unité systématique commune confirmée par une forte similitude qualitative globale entre eux. Ils apparaissent fortement diversifiés puisqu'ils comportent 24 espèces sur 29 espèces de poissons recensées dans la Garonne dans ce secteur (DRAE 1985, Belaud et al. 1989). Ces peuplements ichtyques sont donc uniformisés par le peuplement propre de la Garonne.

La prédominance spécifique des cyprinidés (Tableau 1) semble être la caractéristique générale des trois sites étudies qui se situent dans un secteur de fleuve classee comme une zone "à brèmes" (Huet 1949). Ce sont le gardon, la brème, la rotengle, la bouvière, le carassin, la carpe et la tanche qui constituent les espèces constantes $(C>50 \%)$ (Tableau 2). Ces espèces peuvent alors être considérées comme résidentes du bras mort. Si l'on associe 


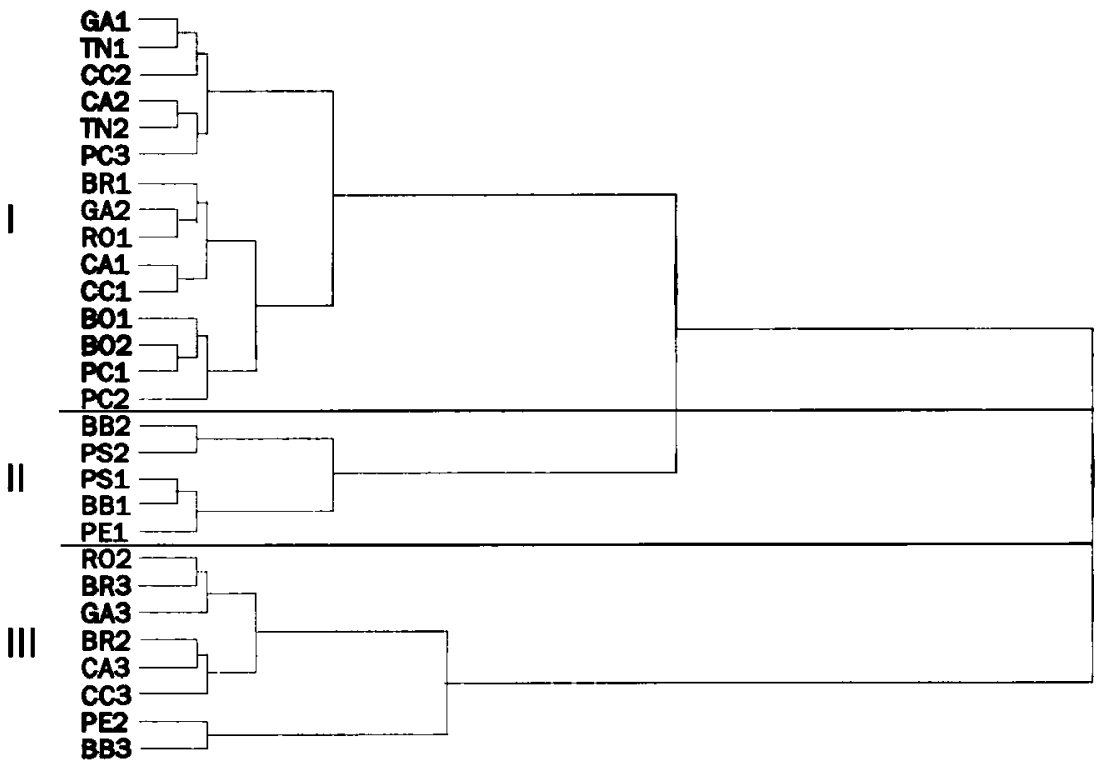

Fig. 5. Dendrogramme de la classification hietarchique des 11 especes subdivisétes en groupes de taille sur les trois premiers facteurs de l'analyse factorielle des correspondances.

Fig. 5. Dendrogram for the hierarchical classification of 11 species, subdivided into size groups, on the first three factors from the correspondence analysis.

à ces cyprinidés le poisson-chat (ictaluridés), la perche (percidés), le black-bass et la perche-soleil (centrarchidés) dont la constance est aussi forte, on constitue ainsi un groupe de 11 espèces résidentes. Ce nombre d'espèces résidentes est suffisamment élevé pour pouvoir donner lieu à une organisation spatiale (Barbault 1981) qui favorise la diversification des niches spatiales.

Malgré leur similitude globale, les peuplements ichtyologiques des trois bras morts présentent certaines différences de structure quantitative qui reflètent les particularités stationnelles. Ces différences de détail paraissent determinées, d'une part par la présence et le type de communication entre le bras mort et le fleuve, permanent et plus large à GF (Ouverture aval à environ $75 \mathrm{~m}$ ) par rapport aux RB (ouverture aval à $17 \mathrm{~m}$ ) et PV $(22 \mathrm{~m}$ ) et, d'autre part, par la structure de l'habitat, plus complexe dans le bras mort " naturel "GF. L'existence de communication aval avec le cours principal et l'hétérogénéité spatiale, donnent lieu à une meilleure richesse spécifique (Stenseth 1980) et à une meilleure productivité piscicole des bras morts (Copp \& Penaz 1988, Scott \& Nielsen 1989). Les présents résultats confirment ces données, du moins pour ce quiconcerne la richesse spécifique.

L'analyse factorielle des correspondances de la structure en classes de taille met en évidence la 


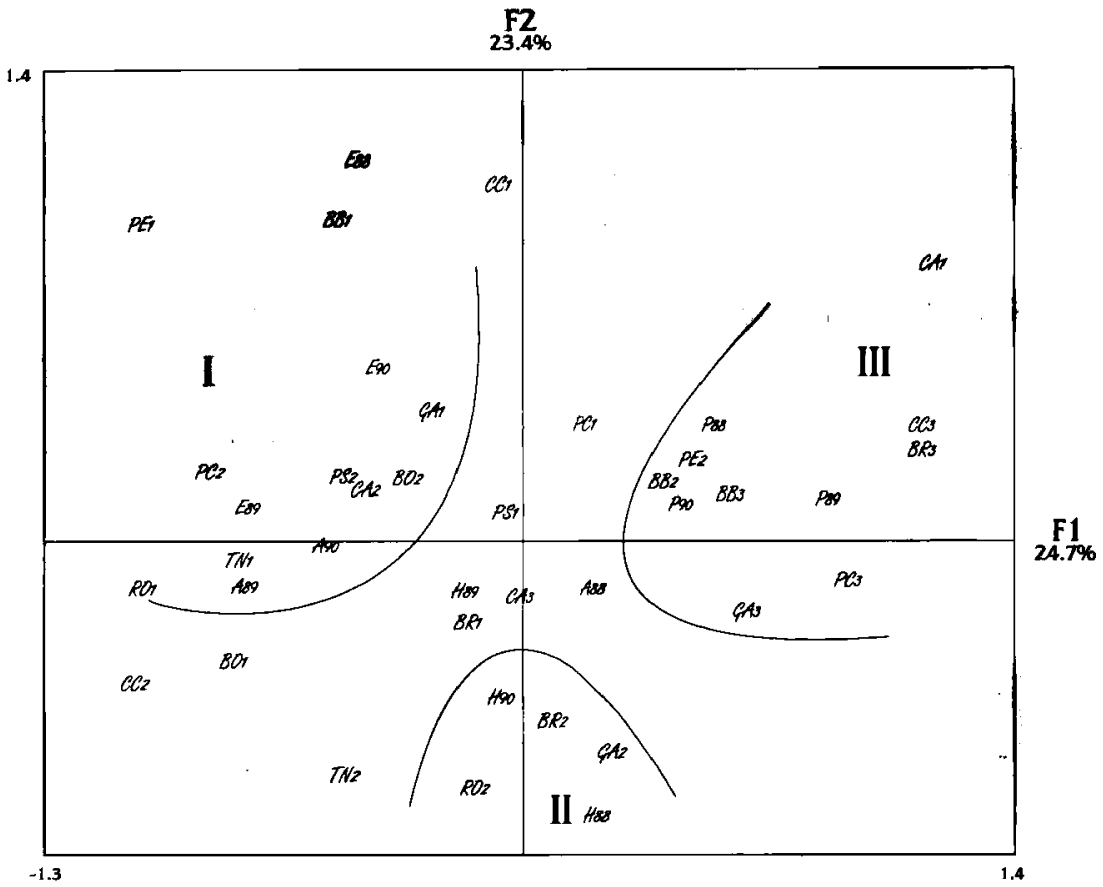

Fig. 6. Analyse factorielle des correspondances. Projection simultanèe des 12 dates d'échantillonnages et des 11 espèces subdivisées en groupes de taille, dans le plan des deux prerniers axes factoriels.

Fig. 6. Correspondence analysis. Simultaneous projection of 12 sampling dates and 11 species, subdivided into size groups, arranged according to the first two major axes.

présence simultanée des plusieurs groupes de tailles (Fig. 2a, 2b, 2c et 2d) correspondant aux différents stades de développement (Tableau 4). C'est un phénomène général chez les espèces grégaires, telles les nombreuses espèces de cyprinidés. Chaque population apparaît comme une mosaïque de bancs différents par leur structure et le nombre d'individus qui les compose. Selon Philippart (1975), ces différences de structure portent sur la taille, le sexe, le stade de maturation sexuelle ou d'autres facteurs. De l'ensemble des espèces examinées, à l'exception de la bouvière et de la perche-soleil, la structure de popu- lation se décompose en définitive en trois groupes ou sous-populations : les juvéniles (individus d'âge $0+$ jusqu'à 1 an), les immatures (de $1+$ à $2^{+}$) et les adultes, âges au-delà de $2+$ ans. La présence simultanée de tous les stades dans les bras morts (cas de la plupart des cyprinidés), traduit une possibilité de sédent arisation complète de ces espèces. La présence de quelques stades seulement, à un moment donné, traduit des phénomènes de migrations (cas des adultes de gardon, brème, carpe, perche et black-bass). Les migrations pourraient être déterminées par des attirances pour certains habitats ou pour certains 


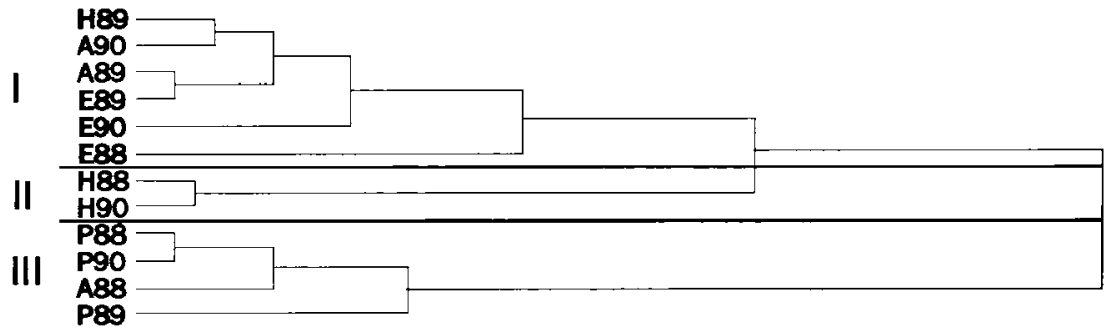

Fig. 7. Dendrogramme de la classification hiérarchique des 12 dates d'échantillonnages sur les trois premiers facteurs de l'analyse factorielle des correspondances.

Fig. 7. Dendrogram for the hierarchical classification of the 12 sampling dates on the first three factors from the correspondence analysis.

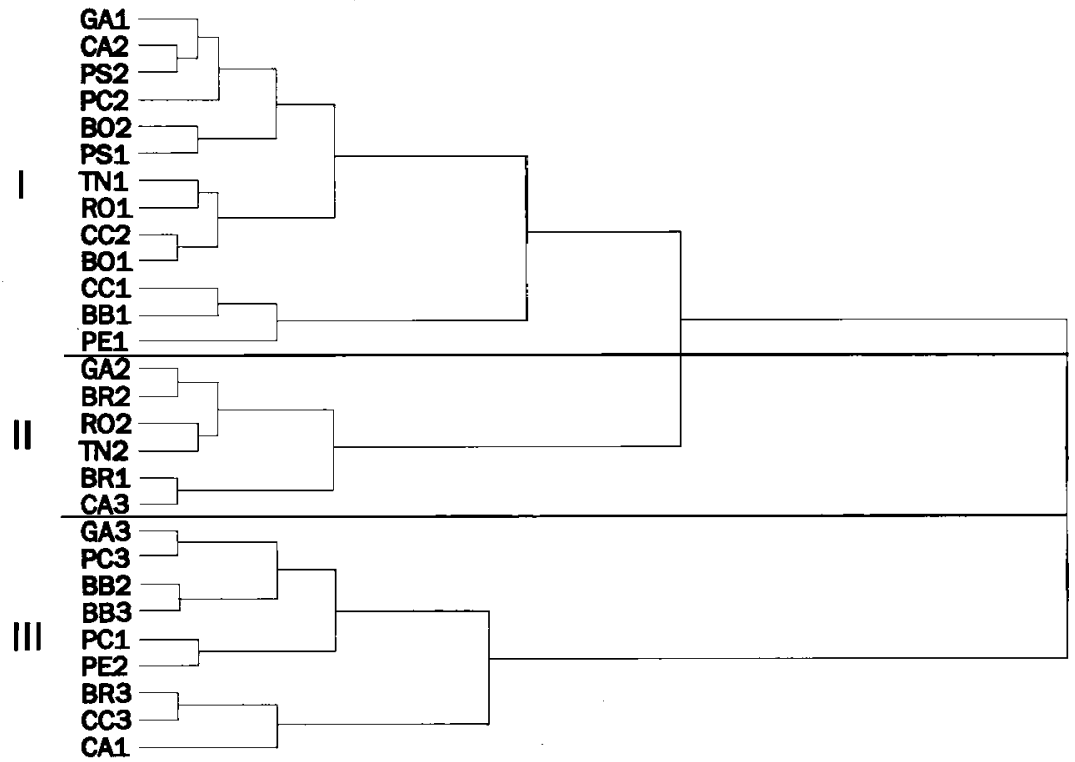

Fig. 8. Dendrogramme de la classification des 11 espèces subdivisées en grcupes de taille sur les trois premiers facteurs de l'analyse factorielle des correspondances.

Fig. 8. Dendrogram for the hierarchical classification of 11 species, subdivided into size groups, on the first three factors from the correspondence analysis. 
groupements interspécifiques (Sheaffer \& Nickum 1986 ; Scott \& Nielsen 1989).

L'analyse factorielle des correspondances montre que l'organisation spatiale de l'ichtyofaune de ces trois bras morts est l'expression des différents composants de l'habitat. L'interaction peuplementhabitats et les influences de l'habitat sont indissociables. La schématisation de ces interactions (Fig. 3), s'est révélée être un moyen intéressant pour évaluer la contribution de chaque groupe d'espèces à la discrimination de l'habitat.

Trois groupes d'habitats ont été isolés sur le plan factoriel F1 $\times$ F2 (Fig. 3) possédant chacun des peuplements qui leur sont propres. Le premier groupe indique la préférence des bouvières et des juvéniles de gardon, carassin, carpe et tanche (cyprinidés) pour les zones littorales peu profondes où se trouve la végétation aquatique de type renoncules et nymphea en bordure des substrats à granulométrie limon-vase. Ces preferanda sont essentiellement caractéristiques du bras mort GF (partie amont) et de la partie aval du bras mort RB. Ce type d'association, commun dans les systèmes lentiques (Vadas 1990), occasionne d'une part la compétition interspécifique et, d'autre part, la compétition alimentaire (Keast 1978a, Mark et al. 1987, Bergman 1990). En revanche, ce comportement grégaire est considéré comme intéressant dans la stratégie de survie face aux prédateurs (Nikolski 1978). La densité de la végétation et la faible luminosité dans ces habitats sont aussi à l'avantage des proies par rapport aux prédateurs.

Les carnassiers de petites tailles (perche-soleil, perche et black-bass) sont essentiellement responsables de la discrimination du second groupe d'habitat. Ces poissons sont présents dans un réseau de végétation aquatique de type phragmites. Comme l'ont souligné Keast (1978b), Prince \& Maughan (1979) et Colle et al. (1989), ces petits carnassiers préfèrent les eaux claires à végétation aquatique semiimmergée car les proies attirées par cette structure y sont abondantes. Le taux de prédation est significativement réduit lorsque la compléxité structurale augmente et le nombre de proies reste constant (Savino \& Stein 1982), mais ce taux de prédation pourrait être maintenu à un niveau élevé si le nombre de proies dans les zones à forte densité de végétation augmentait significativement (Savino \& Stein 1989).
Les adultes (gardon, brème, carassin et perche) caractérisent principalement le groupe III ; ils sont structurés tout d'abord en fonction des rives à pente moyenne ou forte où se trouvent les profondeurs supérieures à $1 \mathrm{~m}$. Puis, sur leur préférence d'habitats, se dégage le type de substrat à prédominance de gravier-galets, représentatif sur les zones plus aval des bras morts PV et GF. Pour ce groupe d'adultes, l'effet des caractéristiques du milieu sur la répartition des individus se hiérarchise différemment d'un bras mort à l'autre. Ceci traduit les fortes potentialités adaptatives, du fait notamment de leur forte mobilité, et l'opportunisme des adultes vis-à-vis d'un milieu lentique soumis à des conditions hydrauliques variables.

L'ichtyofaune de ces trois bras morts permet aussi de mettre en évidence les groupements en fonction des saisons, se traduisant par une évolution saisonnière des effectifs spécifiques. La variabilité saisonnière est nettement perceptible dans la formation de groupes, avec trois grandes tendances (Fig. 6) : recrutement estival et automnal de juvéniles dans les bras morts, prépondérance d'individus immatures de quelques cyprinidés en hiver et arrivée printanière de populations d'adultes.

Les trois différents groupes saisonniers sont à mettre en relation avec les cycles biologiques des espèces et les fluctuations de niveau du fleuve. En accord avec Holland \& Huston (1985), Sheaffer \& Nickum (1986) et Scott \& Nielsen (1989), les dominances estivales et automnales de juvéniles dans les bras morts, ainsi que d'immatures en hiver, montrent le rôle de ce type de milieu comme site propice à la reproduction et aux premiers stades de développement. Ce rôle de nurserie est confirmé par l'entrée d'adultes en phase de maturation et formant un troisième groupe au printemps. La remontée d'adultes dans les bras morts durant cette période de hautes eaux printanières assure donc, d'une part, le recrutement qui maintient un peuplement important et diversifié entre le bras mort et la Garonne (Daget in Welcomme 1979, Van Vooren 1981, Holland \& Sylvester 1983, Scott \& Nielsen 1989) et, d'autre part, le déroulement des processus biologiques de reproduction des poissons (Carrel 1986, Sheaffer \& Nickum 1986, Copp 1989).

L'ensemble des résultats indique que les densités numériques (par 100 E.P.A.) de juvéniles de gardon, rotengle, carpe, tanche et black-bass passent 
de 108 individus en été à 55 en hiver. En revanche, les immatures et les adultes passent de 43 individus à 182. Cette différence permet d'apprécier semiquantitativement l'importance relative du recrutement imputable aux bras morts et qui contribue à l'équilibre des populations piscicoles de la Garonne.

Cette analyse des événements saisonniers est à mettre en parallèle avec le suivi de la qualité physicochimique des eaux (Bengen et al. 1992). Le printemps constitue le point initial de l'hydrobiologie des bras morts, avec la submersion des bras morts et l'alignement de la qualité des eaux sur celle du fleuve. La ponte des cyprinidés dans les bras morts est ensuite avantagée par un réchauffement de l'eau plus rapide dans le bras mort par rapport à la Garonne. L'enrichissement progressif du milieu en éléments fertilisants qui intervient par la suite (Bengen et al. 1992), stimule la productivité de ces milieux (Copp \& Penaz 1988, Scott \& Nielsen 1989), au moment du developpement des juvéniles. L'ampleur de la communication aval entre le bras mort et le fleuve joue un rôle à la fois pour les migrations printanières d'adultes vers le bras mort et pour les principaux apports d'éléments fertilisants provenant du fleuve après les crues printanières. L'ensemble de résultats physico-chimiques (Bengen et al. 1992) et les présents résultats hydrobiologiques des bras morts conduit à classer l'intérêt de chaque zone selon le degré de communication en aval avec le fleuve dans l'ordre : $\mathrm{GF}>\mathrm{PV}>\mathrm{RB}$ et selon leur diversité d'habitats dans l'ordre : GF $>$ PV $>$ RB. Cette analyse permet de conseiller des réaménagements des bras morts en respectant la diversité des biotopes et l'ouverture importante en aval. En revanche, la communication amont, qui s'oppose au réchauffement précoce de l'eau au printemps, est sans intérêt, du moins dans les conditions actuelles de qualité des eaux.

\section{Travaux cilés}

Amoros C. \& Chessel D. 1985. - Les peuplements de cladocères (crustacés) descripteurs de fonctionnement hydrologique des bras morts fluviaux. Annis Limnol, 21 (3) : 227-240.

Badia J. \& Do Chi T. 1976. - Etude cinétique de la structure des populations de Squilla mantis (Crustacea : Stomatopoda) par l'analyse factorielle des correspondances. Mar. Biol., 36 : 159-168.

Beaudelin P. 1987. - Les méfaits des extractions de galets de Garonne. Revue Adour-Garonne, 34 : 10-13.

Belaud A., Bengen D. \& Lim P. 1990. - Approche de la structure du peuplement ichtyologique de six bras morts de la Garonne. Annls Limnol., 26 (1) : 81-90.
Bengen D. 1988. - Etude ichtyologique de bras morts de la Garonne en période hivernale. D.E.A., Univ. Paul-Sabatier, Toulouse III, $28 \mathrm{p}$.

Bengen D., Lim P. \& Belaud A. 1992. - Qualité des eaux de trois bras morts de la Garonne : variabilité spatio-temporelle. Rev. Sci. Eau (sous presse)

Benzécri J.P. \& coll. 1982. - L'analyse des données. . 2. L'analyse des correspondances. Dunod, Paris, 632 p.

Benzécri J.P. \& Benzècri F. 1984. - Pratique de l'analyse des données. 1. Analyse des correspondances et classification. Exposé élémentaire. Dunod, Paris, 456 p.

Bergman E. 1990 . - Effects of roach Rutilus rutilus on two percids, Perca fluviatilis and Gymnocephalus cernua : importance of species interactions for diet shifts. Oikos, $57: 24$ l-249.

Bouvet Y., Cristau-Quost I. \& Thiero Yatabri N. 1982. - Structure et fonctionnement des écosystèmes du Haut-Rhône français. XXII : contribution à l'étude des poissons d'un bras mort, la lône des Pêcheurs. Bull. Soc. Linné. Lyon, 5 : 134147.

Brower J.E. \& Zar J.H. 1977. - Field and laboratory methods for general ecology. Wm. C. Brown Co. Publ., lowa, 194 p.

Carrel G. 1986. - Caracterisation physico-chimique du Haut-Rhône français et de ses annexes : incidences sur la croissance des populations d'alevins. Thèse de doctorat, Univ. Lyon 1,185 p.

Carrel G. \& Juget J. 1987. - La Morte du Sauget, un ancien méandre de Rhône : bilan hydrologique et biogéochimique. Schweiz. Z. Hydrol, 49 (1) : 102.125.

Castella C. \& Amoros C. 1984. - Répartition des characees dans les bras morts du Haut-Rhône et de l'Ain et sa signification écologique. Cryptogamie, Algologie, 5 : 127-139.

Celeux G., Diday E., Govaert G., Lechevallier Y. \& Ralambondrainy H. 1989. - Classification automatique des données. Environnement statistique et informatique. Dunod, Paris, $285 \mathrm{p}$.

Chambert C. 1984. - Déplacement de poissons dans un milieu annexe de grand fleuve : cas des tanches et poissons-chat de la lône des Pêcheurs (Rhône). Rev. fr. Sci. Eau, 3 : 375-394.

Chessel D., Lebreton J.D. \& Prodon R. 1982. - Mesures symétriques d'amplitude d'habitat et de diversite intra-echantillon dans In tableau espèoes-relevés : cas d'un gradient simple. C.R. Acod. Sci., Paris, $295: 83-88$.

Chessel D. \& Débouzie D. 1983. - Analyse des correspondances et écologie : causes et conséquences du succès. Actes XII Coll. Méth. appl. à la Géographie, Besançon.

Clifford H.T. \& Stephenson W. 1975. - An introduction to mumerical classification. Academic Press, Inc., New-York, 229 p.

Colle D.E., Cailteux R.L. \& Shireman J.V. 1989. - Distribution of Florida largemouth bass in a lake after elimination of all submersed aquatic vegetation. N. Amer. J. Fish, Mgt., $9: 213-218$.

Copp G.H. 1987. - Le rôle et le fonctionnement des milieux aquatiques du Haut-Rhône français comme sites de reproduction et de nurserie pour les poissons du fleure. Thèse de doctorat, Univ. Lyon I, $94 \mathrm{p}$.

Copp G.H. 1989. - The habitat diversity and fish reproductive function of floodplain ecosystems. Env. Biol. Fish., $26: 1-27$.

Cross D.G. 1976. - A method of comparing the efficiencies of electric fishing operations. J. Fish Biol., 9 : 261-265.

Décamps H. \& Naiman R.J. 1989. - L'écologie des fleuves. La Recherche, 208 : 310-319.

Diday E., Lemaire J., Pouget J. \& Testu F. 1982. Eléments d'analyse de données. Dunod, Paris, $464 \mathrm{p}$. 
Digby P.G.N. \& Kempton R.A. 1987. - Multivariate analysis of ecological communities. Chapman \& Hall Ltd., London, $206 \mathrm{p}$.

Escofier B. \& Pagès J. 1988. - Anatyses foctorielles simples et multiples. Objectifs, méthodes et interprétation. Dunod, Paris, $241 \mathrm{p}$.

Falta E. 1988. - Suivi écologique de la réouverture des bras morts de la Garonne moyenne. D.A.A., E.N.S.A. Toulouse, $106 \mathrm{p}$.

Foucart T. 1985. - Analyse factorielle. Programmation sur micro-ordinateurs. Masson, Paris, 234 p.

Gascuel D. 1985. - Contribution à l'étude écologique des estuaires du littoral Atlantique français : la faune accompagnatrice de la civelle. Thèse de docteur-ingénieur, Univ. Rennes I, 330 p.

Holland L.E. \& Sylvester J.R. 1983. - Distribution of larval fishes related to potential navigation impacts on the Upper Mississippi River, Pool 7, Trans. Am., Fish. Soc, 112 : 293-301.

Holland L.E. \& Huston M.L. 1985. - Distribution and food habits of young-of-the-year fishes in a backwater lake of the Upper Mississippi River. J. Freshwat. Ecol., 3 : 81-92.

Huet H. 1949. - Aperçu des relations entre la pente et les populations piscicoles des eaux courantes. Rev. Suisse Hydrol., IX : 332-351.

Jacquet-Labrot C. 1986. - Evolution des anciens chenaux du Rhồne et de l'Ain : écologie rétrospective basée sur les restes de cladocères. Thèse de doctorat, Univ. Lyon I, $194 \mathrm{p}$.

Jambu M. 1989. - Exploration informatique et statistique des données. Dunod, Paris, 503 p.

Juget J., Amoros C., Gamulin D., Reygrobellet J.L., Richardot M., Richoux Ph, \& Roux C. 1976. - Structure et fonctionnement des écosystèmes du Haut-Rhône français. II : Etude hydrologique et écologique de quelques bras morts. Premiers résultats, Bull. Ecol., 7 (4) : 479-492.

Juget J. \& Roux A.L. 1982. - Une lône du Rhône, zone humide en position de lisière dans l'espace et dans le temps. Bull. Ecol., 13 (2) : 109-124.

Keast A. 1978a. - Trophic and spatial interrelationships in the fish species of an Ontario temperate lake. Env. Biol. Fish, $3: 7-31$.

Keast A. 1978b. - Feeding interrelations between age-groups of pumpkinseed (Lepomis gibbosus) and comparisons with bluegill (Lepomis macrochirus). J. Fish. Res. Bd. Can., $35: 12-27$.

Koblickaja A.P. 1981 . - Key for identifying young freshwater fishes. Light \& Food industrial Publ. House, Moscow, $208 \mathrm{p}$.

Lamarque P., Therezien Y. \& Charlon N. 1975. - Etude des conditions de la pêche électrique dans les eaux tropicales. Bull. Cent. Etud. Rech. Sci. Biarritz, 10 (3) : 403-554.

Lébart L., Morineau A. \& Fénélon J.P. 1979. - Traitement des données statistiques. Dunod, Paris, $240 \mathrm{p}$.

Legendre L. \& Legendre P. 1979. - Ecologie numérique. Tome 2 : La structure des données écologiques. Masson, Paris et la Presse de l'Université du Québec, 254 p.

Ludwig J.A. \& Reynolds J.F. 1988. - Statistical ecology. A primer on methods and computing. John Wiley \& Sons, Inc. New York, 337 p.

Magurran A.E. 1988. - Ecological diversity and its measurement. Croom Helm Ltd., London, 179 p.
Mark W., Hofer R. \& Wieser W. 1987. - Diet spectra and ressource partitioning in the larvae and juveniles of three species and six cohorts of cyprinids from a subalpine lake. Oecologia, $71:$ 388-396.

Moreau G. \& Legendre L. 1979. - Relation entre habitat et peuplements de poissons : essai de définition d'une méthode numérique pour des rivières nordiques. Hydrobiologia, 67 (1) : 81-87.

Nelva A., Persat H. \& Chessel D. 1979. - Une nouvelle méthode d'étude des peuplements ichtyologiques dans les grands cours d'eau par échantillonnage ponctuel d'abondance. C.R. Acad. Sci., t. 289, sér. D : 1295-1298.

Nelva A. 1985. - Biogéographie, démographie et écologie de Chondrostoma nasus nasus (L., 1758) (Hotu, Poisson, Téléostéen, Cyprinidé). Thèse doct. d'Et at es Sciences, Univ. Lyon 1, 349 p.

Nelva A. 1989. - Structure de population en classes de taille et structure en bancs chez le Hotu Chondrostoma nasus (Pisces, Cyprinidae), dans le Haut-Rhône français. Cybium, 13 (3) : 221-233.

Nikolski G.V. 1978, - The ecology of fishes. T.F.H. Publications, Inc. Ltd., London, 351 p.

Philippart J.C. 1975. - Dynamique des populations de poissons d'eau douce non exploitées. In : Lamotte M. \& Bourlière F. (eds). Problèmes d'écologie : la démographie des populations de vertébrés. Masson, Paris, 292-394.

Prince E.D. \& Maughan O.E. 1979. - Attraction of fishes to tire reefs in Smith Mountain Lake, Virginia. In : Johnson D.L. \& Stein R.A. (eds). Response of fish to habitat structure in standing water, North Central Division, Amer. Fish. Soc. Spec. Publ. 6, Bethesda, 19-25.

Roux M. 1985. - Algorithmes de classification. Masson, Paris, $151 \mathrm{p}$.

Savijo J.F. \& Stein R.A. 1982. - Predator-prey interaction between largemouth bass and bluegills as influenced by simulated, submersed vegetation. Trans. Amer. Fish. Soc, 111 : 255-266

Savino J.F. \& Siein R.A. 1982 . - Behavior of fish predators and their prey : habitat choice between open water and dense vegetation. Env. Biol. Fishes, 24 (4) : 287-293.

Scott M.T. \& Nielsen L.A. 1989. - Young fish distribution in backwaters and main-channel borders of the Kanawha River, West Virginia. J. Fish Biol., 35 (suppl. A) : 21-27

Sheaffer W.A.\& Nickum J.G. 1986. - Backwater areas as nursery habitats for fishes in Pool 13 of Upper Mississippi River. Hydrobiologia, 136 : 131-140.

Spillmann C.J. 1961. - Poissons d'eau douce. Faune de France. Editions Paul le Chevalier, 303 p.

Stenseth N.C. 1980 . - Spatial heterogeneity and population stability : some evolutionary consequences. Oikos, $35: 165-184$.

Vadas Jr R.L. 1990. - The importance of omnivory and predator regulation of prey in freshwater fish assemblages of North America. Env. Biol. Fishes, 27 : 285-302.

Van Vooren A, 1981. - Relative utilization of Mississippi river habitats as nursery areas. Iowa Cons. Comm. Proj., 81-IIIC-11: 103-122.

Vibert R. 1968. - Application de l'électricité à la biologie et à l'aménagement des pêches continentales. Acte Symp. FAO, Belgrade, Ed. INRA, Paris, 276 p.

Welcomme R.L. 1979. - Fisheries ecology of floodplain rivers. Longman, London and New-York, $317 \mathrm{p}$.

Zalewski M. 1986. - Factors affecting the efficiency of electrofishing in rivers. Hydrobiology, $27: 56-66$. 